\title{
Stress Exacerbates Neuron Loss and Cytoskeletal Pathology in the Hippocampus
}

\author{
B. Stein-Behrens, ${ }^{1}$ M. P. Mattson, ${ }^{2}$ I. Chang, ${ }^{1}$ M. Yeh, ${ }^{1}$ and R. Sapolsky' \\ 'Department of Biological Sciences, Stanford University, Stanford, California 94305 and ${ }^{2}$ Sanders-Brown Research Center \\ on Aging and Department of Anatomy and Neurobiology, University of Kentucky Medical Center, Lexington, Kentucky \\ 40536-0230
}

\begin{abstract}
Glucocorticoids (GCs), the adrenal steroids secreted during stress, endanger the hippocampus, compromising its ability to survive neurological insults. GCs probably do so by disrupting energetics in the hippocampus, thus impairing its ability to contain damaging fluxes of excitatory amino acids and calcium. Superficially, these observations suggest that stress itself should also exacerbate the toxicity of neurological insults. However, most studies have involved unphysiologic GC manipulations, limiting speculations about the endangering effects of stress. In this study, rats were infused with the excitotoxin kainic acid (KA) after either having been adrenalectomlzed and replaced with a range of physiologic concentrations of GCs, or having been stressed intermittently. We observed that within the CA3 region, increasing CORT concentrations exacerbated the KA-induced neuron loss, the extent of tau immunoreactivity, and of spectrin proteolysis. The transitions from low to high basal GC concentrations and from high basal to stress GC values were both associated with significant exacerbation of neuron loss and tau immunoreactivity; the extent of spectrin proteolysis was less sensitive to increments in GCs. As would be expected from these data, exposure to intermittent stress prior to KA infusion also exacerbated neuron loss, tau immunoreactivity, and spectrin proteolysis in CA3. Thus, physiological elevations of GCs, and stress itself, can exacerbate hippocampal neuron loss and the attendant degenerative markers following an excitotoxic insult. Of significance, seizure and hypoxia-ischemia provoke considerable GC stress responses, which may thus worsen the resultant damage. Furthermore, a number of neuropsychiatric disorders, as well as aging, are associated with elevated basal GC concentrations, which may endanger the hippocampus in the event of neurological insult.
\end{abstract}

[Key words: stress, hippocampus, glucocorticoids, corticosterone, spectrin, tau, cytoskeleton]

Glucocorticoids (GCs), the adrenal steroids released during stress, can damage the hippocampus, a principal neural target site for

\footnotetext{
Received Nov, 8, 1993; revised Jan. 20, 1994; acceptcd March 2, 1994.

Funding was made possible by grants to R.S. from the NIH, the Alzheimer's Association, and the Adler Foundation, and to M.P.M. from the NIH, the Metropolitan Life Foundation, and the Alzheimer's Association. We thank Dr. K. Beck for technical assistance. We thank P. Davies and K. Kosik for providing antibodies ALZ-50 and 5E2, respectively.

Correspondence should be addressed to Dr. R. Sapolsky at the above address. Copyright (C) 1994 Society for Neuroscience $0270-6474 / 94 / 145373-08 \$ 05.00 / 0$
}

GCs (Landfield et al., 1981; Sapolsky et al., 1985, 1990; Meaney et al., 1988; Woolley et al., 1990; Talmi et al., 1993). Similarly, stress itself can damage the hippocampus (Uno et al., 1989; Kerr et al., 1991; Mizoguchi et al., 1992; Watanabe et al., 1992). Glucocorticoids also exacerbate hippocampal neuron loss during insults such as hypoxia-ischemia (Sapolsky and Pulsinelli, 1985; Koide et al., 1986; Hall, 1990; Morse and Davis, 1990; Miller and Davis, 1991), seizure (Sapolsky, 1985; Theoret et al., 1985; Stein and Sapolsky, 1988), hypoglycemia or antimetabolite exposure (Sapolsky, 1985; Tombaugh et al., 1992), or exposure to various neurotoxins (Johnson et al., 1989; Hortnagl et al., 1993).

Such GC-induced endangerment of the hippocampus appears to involve exacerbation of the well-characterized cascade by which excessive synaptic concentrations of excitatory amino acid (EAA) neurotransmitters produce toxic levels of free cytosolic calcium. (1) Numerous insults that damage via this EAA calcium cascade are worsened by GCs. (2) GCs exacerbate extracellular accumulation of EAAs in the hippocampus following seizure (Stein-Behrens et al., 1992), and inhibit EAA uptake by cultured hippocampal neurons and glia (Virgin et al., 1991; Chou et al., 1994). (3) GC endangerment of the hippocampus is eliminated by blockade of the NMDA receptor (Armanini et al., 1990). (4) GCs cause EAA-induced rises in cytosolic calcium in hippocampal neurons to be larger and more persistent (Elliott and Sapolsky, 1992, 1993). (5) GCs exacerbate the calciumdependent proteolysis of spectrin and antigenic alterations in tau immunoreactivity following EAA exposure (Elliott et al., 1993). These varied GC actions are generally reversed by coincident supplementation with glucose or mannose, suggesting that the GC endangerment of the hippocampus is energetic in nature. As a possible root of this energetic endangerment, GCs decrease glucose uptake in hippocampus and in cultured hippocampal neurons and glia by approximately $30 \%$ (Kadekaro et al., 1988; Horner et al., 1990; Virgin et al., 1991; Freo et al., 1992).

These studics suggest that stress, via GC secretion, can have a deleterious impact upon the severity of varied neurological insults to the hippocampus. However, this supposition about the physiological relevance of GC neuroendangerment has not really been tested. This is because the studies cited either relied upon in vitro preparations or, in the case of in vivo studies, involved unphysiologic GC manipulations (the use of synthetic GCs, exposure to supraphysiologic GC levels or patterns in experimental groups, or complete elimination of GCs by adrenalectomy in control groups). In the present study we show that GCs exacerbate kainate (KA)-induced neuron loss, tau im- 


$\begin{aligned} & \text { Table 1. Circulating corticosterone concentrations generated by } \\
& \text { pellets of varying corticosterone : cholesterol ratios }\end{aligned}$
\begin{tabular}{lc} 
Percent CORT in pellet & CORT concentration $(\mu \mathrm{g} / \mathrm{dl})$ \\
\hline 0 & Below detection $(1 \mu \mathrm{g} / \mathrm{dl})$ \\
15 & $6.0 \perp 0.8$ \\
60 & $14.1 \pm 1.3$ \\
100 & $21.9 \pm 1.2$ \\
$5 \mathrm{mg}$ injection daily & $28.0 \pm 2.5$ \\
\hline
\end{tabular}

munoreactivity, and spectrin breakdown in the hippocampus in a dose-dependent manner within the whole physiological range. More importantly, we show that stress alone has the same deleterious effects.

\section{Materials and Methods}

Animals and materials. Subjects were male Sprague-Dawley rats (Simonsen, Gilroy, CA) weighing 300-350 gm and housed on a 12:12 hr light/dark cycle with lights on at 0700 . Animals were given food and water ad libitum.

Corticosterone (CORT; the predominant GC of rats), metyrapone, $\mathrm{KA}$, urethane, and paraformaldehyde were purchased from Sigma (St. Louis, MO).

Glucocorticoid manipulations. In experiments using CORT pellets, rats were adrenalectomized under ether anesthesia, and a $100 \mathrm{mg}$ pellet with the indicated ratio of CORT to cholesterol was implanted subcutaneously (Akana et al., 1986). Rats were given $3 \mathrm{~d}$ for hormone levels to stabilize prior to experimentation. A blood sample was taken during microinfusion for measurement of circulating COKT levels. In the indicated group of rats, even higher CORT concentrations were generating by injecting rats with $5 \mathrm{mg}$ CORT/d (s.c., in peanut oil); the final such injection occurred $2 \mathrm{hr}$ prior to the beginning of the microdialysis. A blood sample was taken at the beginning of the microinfusion experiment for measurement of circulating CORT levels.

In experiments involving stress, unstressed rats were undisturbed and, to avoid a GC stress response to the microinfusion surgery, were injected 30 min prior to microinfusion with the adrenocortical steroidogenesis inhibitor metyrapone (2-methyl-1,2-di-3-pyridyl-1-propanone; $200 \mathrm{mg}$ / $\mathrm{kg} \mathrm{BW}$, s.c., in $1.5 \mathrm{ml}$ saline); metyrapone blocks GC secretion, even in response to major stressors (Stein and Sapolsky, 1988). Stressed rats were exposed to a variety of stressors for $3 \mathrm{~d}$. Each day, they were placed in a $4^{\circ} \mathrm{C}$ room for $12 \mathrm{hr}$. During the remaining $12 \mathrm{hr}$, they were exposed to a different stressor every $2 \mathrm{hr}$, including ether exposure, $30 \mathrm{~min}$ of restraint in a tube, $30 \mathrm{~min}$ with the cage on a rotator plate, and intermixing of animals between social groupings (i.e., placing the rats in a new grouping once per day). Stressors were varied to avoid habituation. Restraint stress typically generates circulating CORT values in the 30$40 \mu \mathrm{g} / \mathrm{dl}$ range in our hands, whereas the other stressors produce values in the $15-25 \mu \mathrm{g} / \mathrm{dl}$ range (Sapolsky et al., 1984, 1985). Anesthetization for microdialysis occurred immediately after the final stressor, such that the kainic acid microinfusion camc approximately $2 \mathrm{hr}$ after the end of that stressor.

Microinfusion. Rats were anesthetized with ether and microinfused stereotaxically in the dorsal hippocampus $(\mathrm{D} / \mathrm{V}+4.0, \mathrm{~L} / \mathrm{M} \pm 2.1, \mathrm{~A} / \mathrm{P}$ -3.0 from lambda). One side was injected with KA $(0.07 \mu \mathrm{g}$ in $1 \mu \mathrm{l}$ PBS) while PBS was infused into the contralateral hippocampus as a control.

Tau immunocytochemistry and Nissl staining. These methods were similar to those used in our previous study (Elliot et al., 1993). Three hours after microinfusion, rats were injected with $20 \%$ urethane and were perfused intracardially with a $0.1 \mathrm{~m} \mathrm{PBS} / .05 \%$ heparin solution followed by $4 \%$ paraformaldehyde. Brains were cryoprotected and 30 $\mu \mathrm{m}$ coronal sections were cut. Free-floating sections were incubated at room temperature for $1 \mathrm{hr}$ in PBS containing 0.2\% Triton X-100 and $0.015 \%$ nonimmune horse serum. Sections were incubated overnight in PBS/Triton/nonimmune serum containing a primary antibody. Two different antibodies that recognize tau were used in the present study: Alz-50, a mouse monoclonal raised against homogenate of Alzheimer's disease brain (Wolozin et al., 1986), was a gift from Dr. P. Davies; 5E2,

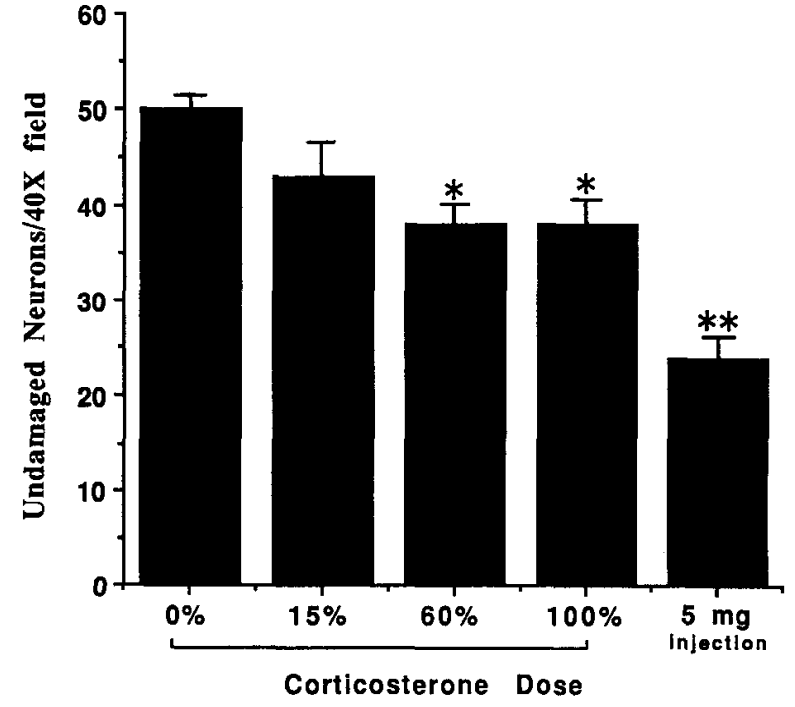

Figure 1. Effect of CORT dose on KA-induced hippocampal injury. Undamaged neurons were counted in CA3 in rats killed $3 \mathrm{hr}$ after KA injection into the hippocampus; rats were adrenalectomized and replaced with indicated amounts of CORT. $n=4-6$ rats. The overall effect of increasing CORT dose was highly significant $[p<0.006$ by ANOVA; $F(19)=6.241 .{ }^{*}, p<0.05$ compared to value for $0 \% \mathrm{CORT}$; ${ }^{* *}, p<0.001$ compared to value for $0 \%$ CORT, and $p<0.01$ compared to value for $100 \%$ CORT (Scheffe test).

a mouse monoclonal raised against fetal human tau, which immunostains neurofibrillary tangles (Joachim et al., 1987; Kosik et al., 1988), was provided by Dr. K. S. Kosik; monoclonal MAP2 antibody (clone AP20) was purchased from Sigma. Antibody dilutions were, for Alz$50,1: 10 ; 5 E 2,1: 200 ;$ and MAP2, 1:1000. Following exposure to primary antibodies, the sections were processed using an anti-mouse Vectastain $\mathrm{ABC}$ biotin-avidin-peroxidase kit with diaminobenzidine tetrahydrochloride as a substrate. Negative controls consisted of eliminating the primary antibodies from the procedure. Sections from brains of control and CORT-treated rats were processed in parallel. Sections were photographed under bright-field optics using the same exposure conditions and on the same roll of film, and prints of each negative were prepared using identical conditions. Counts of immunoreactive neuronal somata were made in the entire extent of region CA3 (counts were made in four sections/brain).

Coronal sections $(30 \mu \mathrm{m})$ were stained with cresyl violet using the Nissl method. Numbers of undamaged Nissl-positive cells within three adjacent $40 \times$ microscopic fields in a portion of the pyramidal cell layer of area CA 3 were counted in each section, and these numbers were used as an indicator of the number of viable neurons present. Neurons with a rounded cell body and a visible nucleolus were considered undamaged, while Nissl-positive cells with a crenated cell body in which the nucleolus was not discernable were considered damaged (see Fig. 4). Cell counts were made (without knowledge of the experimental treatment of the section) within the region of area CA3 extending from a point just below the apex of the lateral bend in the pyramidal cell layer to a point directly ventral to the most lateral extension of the upper limb of the dentate granule cell layer.

Spectrin analysis. Rats were decapitated $6 \mathrm{hr}$ postmicroinfusion. The hippocampi were rapidly dissected and placed in cold dissection buffer (0.32 $\mathrm{m}$ sucrose, $10 \mathrm{~mm}$ Tris-HCl, $2 \mathrm{~mm}$ EDTA, $1 \mathrm{~mm}$ EGTA, $0.1 \mathrm{~mm}$ leupeptin, $1 \mu \mathrm{g} / \mathrm{ml} n$-tosyl-L-phenylalanine chloromethyl ketone, $\mathrm{pH}$ 7.4). Each hippocampus was homogenized in $500 \mu \mathrm{l}$ of dissection buffer. An aliquot was added to $1 / 3$ vol of $3 \times$ sodium dodecyl sulfate-polyacrylamide gel electrophoresis (SDS-PAGE) sample buffer $(150 \mathrm{~mm}$ Tris- $\mathrm{PO}_{4}, 6 \%$ SDS, $10 \%$ glycerol, $3 \% \beta$-mercaptoethanol, $\mathrm{pH} 6.8$ ) and boiled for $5 \mathrm{~min}$. A second aliquot was removed for protein analysis by the Bradford method. The procedure for SDS-PAGE, elcctrophoretic transfer, immunodetection and densitometric quantification were as described previously (Elliott et al., 1993).

Plasma CORT determination. CORT concentrations were determined by RIA using a highly specific antibody (B3-163, Endocrine 


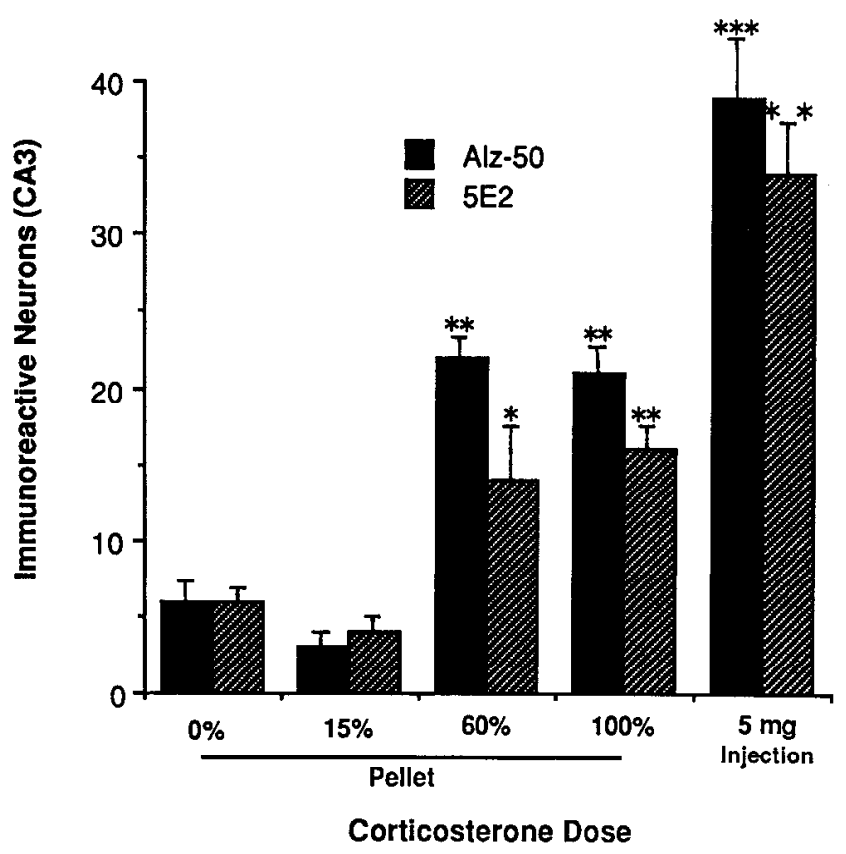

Figure 2. Effect of CORT dose on tau immunoreactivity $3 \mathrm{hr}$ after $\mathrm{KA}$ injection. Brains were processed for immunolocalization of tau immunoreactivity using antibodies $5 \mathrm{E} 2$ and $\mathrm{Alz}-50 ; n=4-6$ rats. The overall effect of increasing CORT dose was highly significant [Alz-50, $p<0.0001, F(49)=51.3 ; 5 \mathrm{E} 2, p<0.0001, F(49)=32.8] . *, p<0.05$ compared to values for $0 \%$ and $15 \%$ CORT; ${ }^{* *}, p<0.01$ compared to values for $0 \%$ and $15 \%$ CORT; ${ }^{* * *}, p<0.001$, compared to values for $0 \%$ and $15 \%$ CORT, $p<0.01$ compared to values for $60 \%$ and $100 \%$ CORT (Scheffe test).

Sciences, Tarzana, CA) and ${ }^{3} \mathrm{H}-\mathrm{CORT}$ tracer as described previously (Gwosdow-Cohen et al., 1982). Coefficients of variation within and between assays were less than $10 \%$. The minimal detectable level of CORT was $0.95 \mu \mathrm{g} / \mathrm{dl}$.

Data analysis. Statistical analyses included ANOVA to determine overall effects in CORT dose-response studies and either paired $t$ test or ANOVA followed by Scheffe's post hoc test for pairwise comparisons between treatment groups.

\section{Results}

CORT/cholesterol pellets produced circulating CORT concentrations that varied as a function of the percentage CORT in each pellet (Table 1): $0 \%$ pellets produced CORT concentrations roughly corresponding to those normally seen during the circadian trough; $15 \%$ pellets produced values mimicking the midbasal range; $60 \%$, mimicking the circadian peak; $100 \%$, mimicking moderate stress; and the $5 \mathrm{mg}$ injection, mimicking concentrations produced in response to a substantial stressor.

Metyrapone-treated rats had circulating CORT values in the basal range $(12.8 \pm 0.8 \mu \mathrm{g} / \mathrm{dl})$, despite undergoing the stressor of stereotaxic surgery. In contrast, CORT values in stressed rats, taken at the same time, were $25.4 \pm 2.5 \mu \mathrm{g} / \mathrm{dl}$.

Kainate-induced damage to the $\mathrm{CA} 3$ region of the hippocampus, as quantified by cresyl violet staining, was exacerbated by physiological concentrations of CORT in a dose-dependent manner (Fig. 1). The transition from exposure to low basal to high basal CORT concentrations (i.e., from $0 \%$ to $60 \%$ pellets) produced a significant increase in damage, while the transition to the substantial stress range (i.e., the $5 \mathrm{mg}$ injection) caused a further worsening of neuron loss.
Table 2. Extent of post-KA spectrin proteolysis as a function of circulating CORT concentrations, or of exposure to stress

\begin{tabular}{ll} 
& $\begin{array}{l}\text { Spcctrin } \\
\text { proteolysis }\end{array}$ \\
\hline CORT manipulation & \\
$0 \%$ pellet & $1.5 \pm 0.6$ \\
$15 \%$ or $60 \%$ pellet & $1.8 \pm 0.8$ \\
$100 \%$ pellet or injection & $4.7 \pm 1.2$ \\
Stress manipulation & \\
Unstressed & $1.7 \pm 0.2$ \\
Stressed & $2.4 \pm 0.2$
\end{tabular}

Spectrin proteolysis indicates the percentage of spectrin recognized on an immunoblot in the breakdown from (i.e., the 155 and $150 \mathrm{kDa}$ products), relative to intact spectrin (see Elliott et al., 1993, for methods). $n=16$ rats/group for the CORT manipulation experiment and $n=5$ rats/group for the stress experiment. In the CORT manipulation experiment $p<0.05$ by ANOVA; proteolysis in the $100 \%$ pellet or injection group differed from the extent of proteolysis in the $0 \%$ pellet group ( $p<0.05$, Scheffe post hoc test). In the stress experiment, the two groups differed at the 0.05 level of significance by paired $t$ test.

Tau immunoreactivity in the CA3 region increased significantly with increasing CORT concentrations (Fig. 2). The transition from low to high basal CORT concentrations (from 0\% to $60 \%$ pellets) caused a significant increase, while the transition to the substantial stress range (i.e., the $5 \mathrm{mg}$ injection) caused a further increase in immunoreactivity.

Kainate-induced spectrin proteolysis was also worsened by increasing CORT concentrations (Table 2); the transition from zero CORT concentrations to those in the basal range did not worsen proteolysis, whereas a further increase in CORT concentrations into the stress range exacerbated proteolysis.

Stress augmented KA actions in a manner similar to that of CORT exposure. Kainate caused significant neuron loss in the CA3 and hilar regions; stress exacerbated the toxicity of KA in

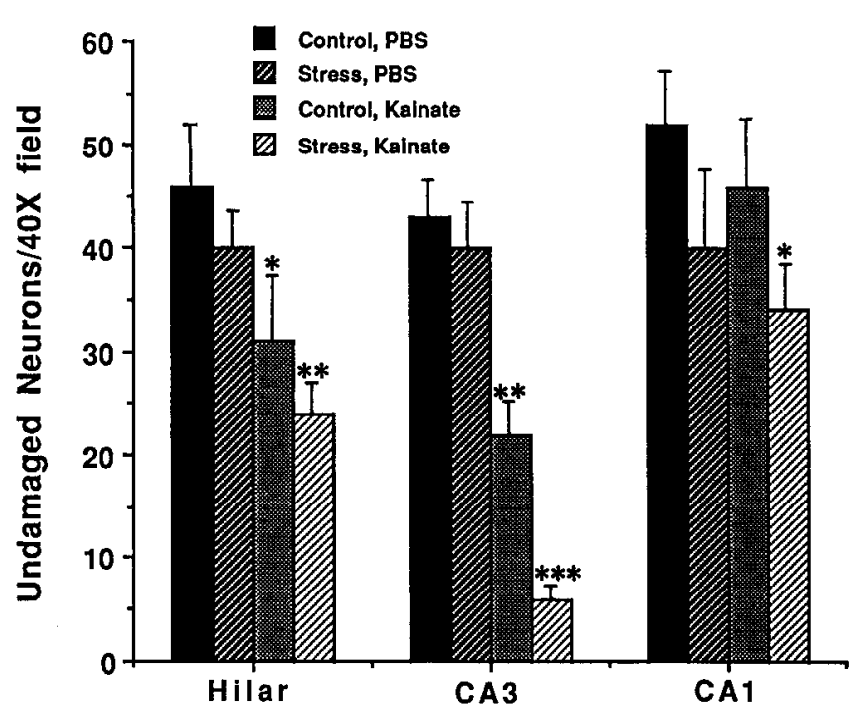

Figure 3. Stress exacerbated hippocampal injury $3 \mathrm{hr}$ after KA injection. Undamaged neurons were counted in the hilar region, $\mathrm{CA} 3$, and $\mathrm{CA} 1$ in control (unstressed, metyrapone-treated) and stressed rats. $n=$ 12 rats/group; data pooled from two experiments. *, $p<0.05$ compared to corresponding value for PBS-injected hippocampus in control rats; ${ }^{* *}, p<0.005$ compared to values for PBS-injected hippocampi in control or stressed rats; ${ }^{* * *}, p<0.006$ compared to corresponding valuc for KA-injected hippocampi in unstressed control rats (Scheffe test). 

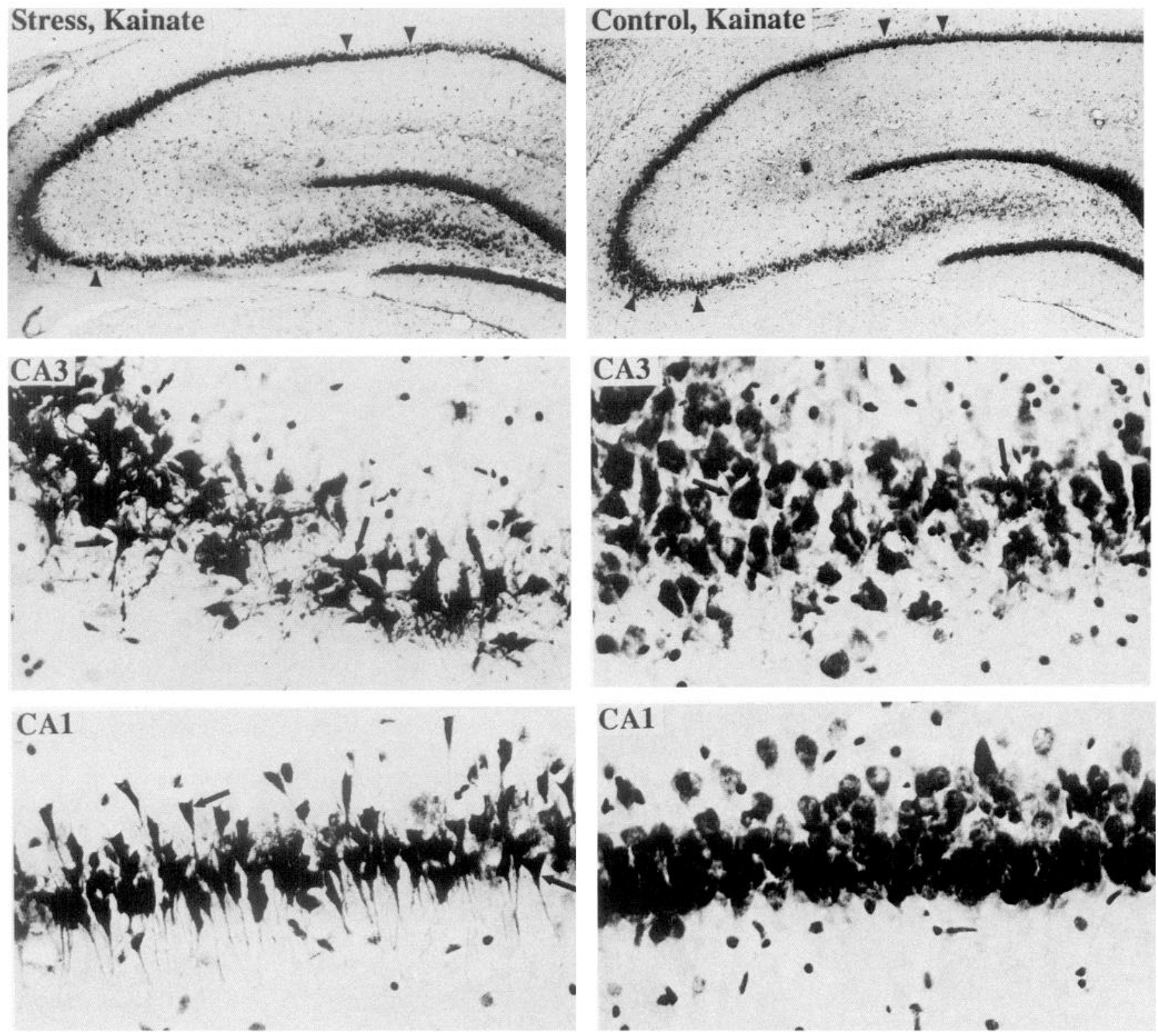

Figure 4. Excitotoxic neuronal damage in the hippocampus of stressed rats is greater than in unstressed animals: cresyl violet-stained coronal sections of hippocampus from stressed KA-injected (left) and control KA-injected (right) rats. The regions of CA3 and CA1 lying between the arrowheads in the low-magnification micrographs $($ top; $40 \times)$ are shown at higher magnification $(400 \times)$ in the middle and bottom micrographs. Neurons are more severely damaged in CA3 of the stressed rat. Damage is also seen in region CA1 of the stressed rat; note the crenated cell bodies compared to the rounder neuronal somate seen in CA1 of the control rat.

the $\mathrm{CA} 3$ region, as compared with unstressed, $\mathrm{KA}$-injected rats (Figs. 3,$4 ; p<0.01)$. Interestingly, neuronal damage was evident in region CA1 of a small percentage of stressed animals ( 3 of 12 animals; e.g., Fig. 4), but was never observed in unstressed animals $(n=12)$, or in animals receiving CORT at any dose $(n$ =46). Microtubule-associated protein 2 (MAP2) is a dendritic protein that is very sensitive to calcium-mediated proteolysis (Johnson et al., 1991). Kainic acid caused a reduction in MAP2 immunoreactivity in the molecular layer of CA3, and this loss of MAP2 immunoreactivity was exacerbated in the stressed rats (Fig. 5). In addition, the number of CA3 neurons immunoreactive with antibodies $\mathrm{Alz}-50$ and $5 \mathrm{E} 2$ was significantly increased in the KA-injected hippocampus of stressed animals compared to controls (Fig. 6; $p<0.01$ for 5E2 and $p<0.05$ for Alz-50). Examples of Alz-50 immunoreactivity in KA-injected hippocampi from stressed and control animals are shown in Figure 7. The section from the stressed animal is from a case where damage to CA1 neurons was observed in Nissl-stained sections (see Fig. 4). Many neurons in the region of neuronal injury in $\mathrm{CAl}$ were $\mathrm{Alz}-50$ immunoreactive, indicating that (as in region CA3) there is a strong correlation between neuronal damage assessed by Nissl-stain and tau immunoreactivity. Finally, stressed, KA-infused rats had significantly more spectrin proteolysis than did unstressed, KA-infused rats (Table 2).

\section{Discussion}

As reviewed in the introductory remarks, sufficient exposure to GCs can directly damage the hippocampus, and a number of 

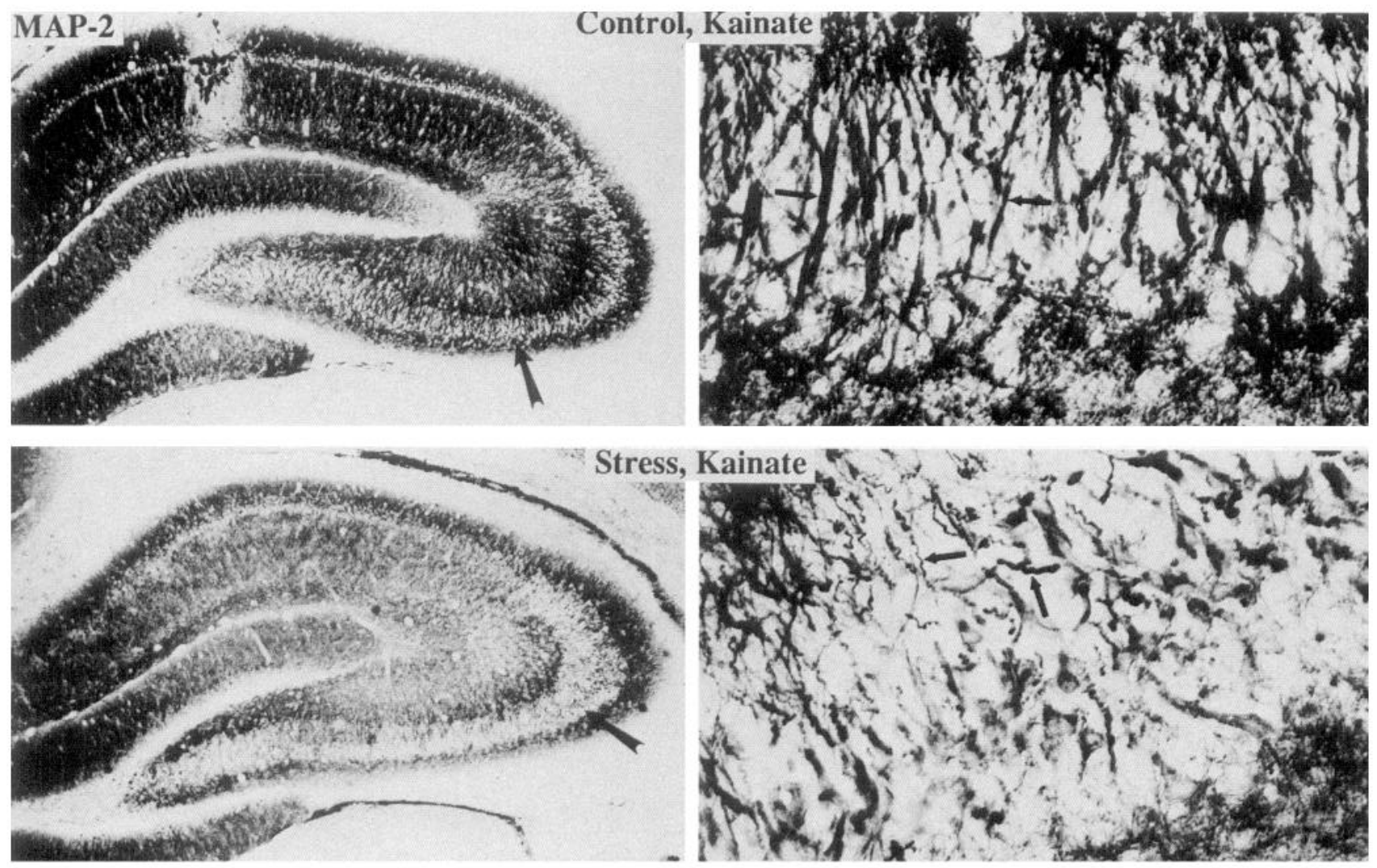

Figure 5. Stress exacerbates KA-induced reduction in MAP-2 immunoreactivity in the hippocampus: MAP-2 immunoreactivity in coronal sections of KA-injected hippocampi from control and stressed rats. The micrographs at the right are higher-magnification $(400 \times)$ views of the region of CA3 marked by the arrow in the lower-magnification micrographs $(l e f t ; 40 \times)$. Note the reduced level of MAP-2 immunoreactivity in region CA3 of the stressed tissue relative to the control. Also note that many neurites in region CA 3 of the stressed tissue exhibit a tortuous "curly" appearance (arrows in high-magnification micrograph) compared to neurites in the control animal, which are generally straight. These micrographs are representative of results obtained in eight control and eight stressed rats.

reports have indicated that sustained stress can as well. GCs can also endanger (i.e., impair the capacity of the hippocampus to survive varied neurological insults), and probably do so by disrupting hippocampal energetics, thereby impairing the capacity of the hippocampus to contain damaging fluxes of EAAs and calcium. Implicit in the interest in the latter findings is the question of whether stress itself can endanger hippocampal neurons and exacerbate the toxicity of neurological insults to the structure. However, as noted, few of the initial studies in this area made answering that question possible, because of the unphysiological nature of the GC manipulations. More recent evidence has emerged that stress itself can exacerbate some of the facets of neuronal dysfunction thought to contribute to excitotoxic neuron death. For example, stress elevates extracellular EAA concentrations in the hippocampus (Moghaddam, 1993), and augments hippocampal metabolism in an NMDA-dependent fashion (Krugers et al., 1992). Our present data indicate that stress also exacerbates excitotoxin-induced accumulation of tau immunoreactivity, and spectrin and MAP2 proteolysis. These findings imply that stress should indeed exacerbate excitotoxic neuron loss in the hippocampus. The present data also show this explicitly (Figs. 3-5); to our knowledge, this is a first such demonstration.

The particular neurodegenerative markers exacerbated by CORT and stress were accumulation of tau antigenicity, loss of MAP2 immunoreactivity, and proteolysis of spectrin. Based upon previous data, it is very likely that all three of these cy- toskeletal alterations were due to excessive elevations of intracellular calcium levels induced by KA and exacerbated by CORT. Previous cell culture studies demonstrated that excitatory amino acids and calcium influx elicit antigenic and biochemical alterations in tau similar to those seen in neurofibrillary tangles

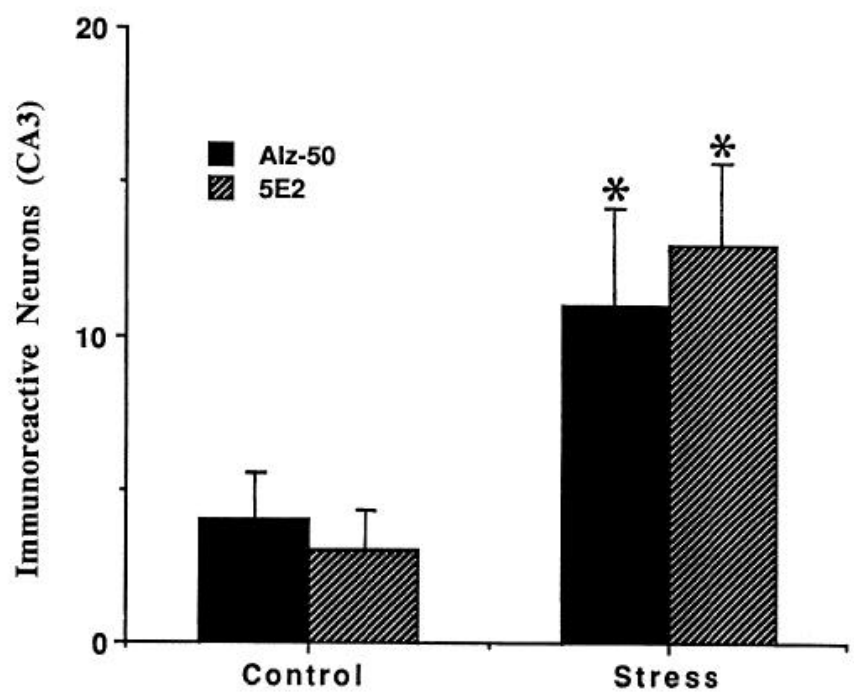

Figure 6. Effect of stress on tau immunoreactivity $3 \mathrm{hr}$ after unilateral $\mathrm{KA}$ injection in control or stressed rats ( $n=8$ rats/group). ${ }^{*} p<0.05$ (Alz-50) or $p<0.01$ (5E2) compared to control (paired $t$ test). 

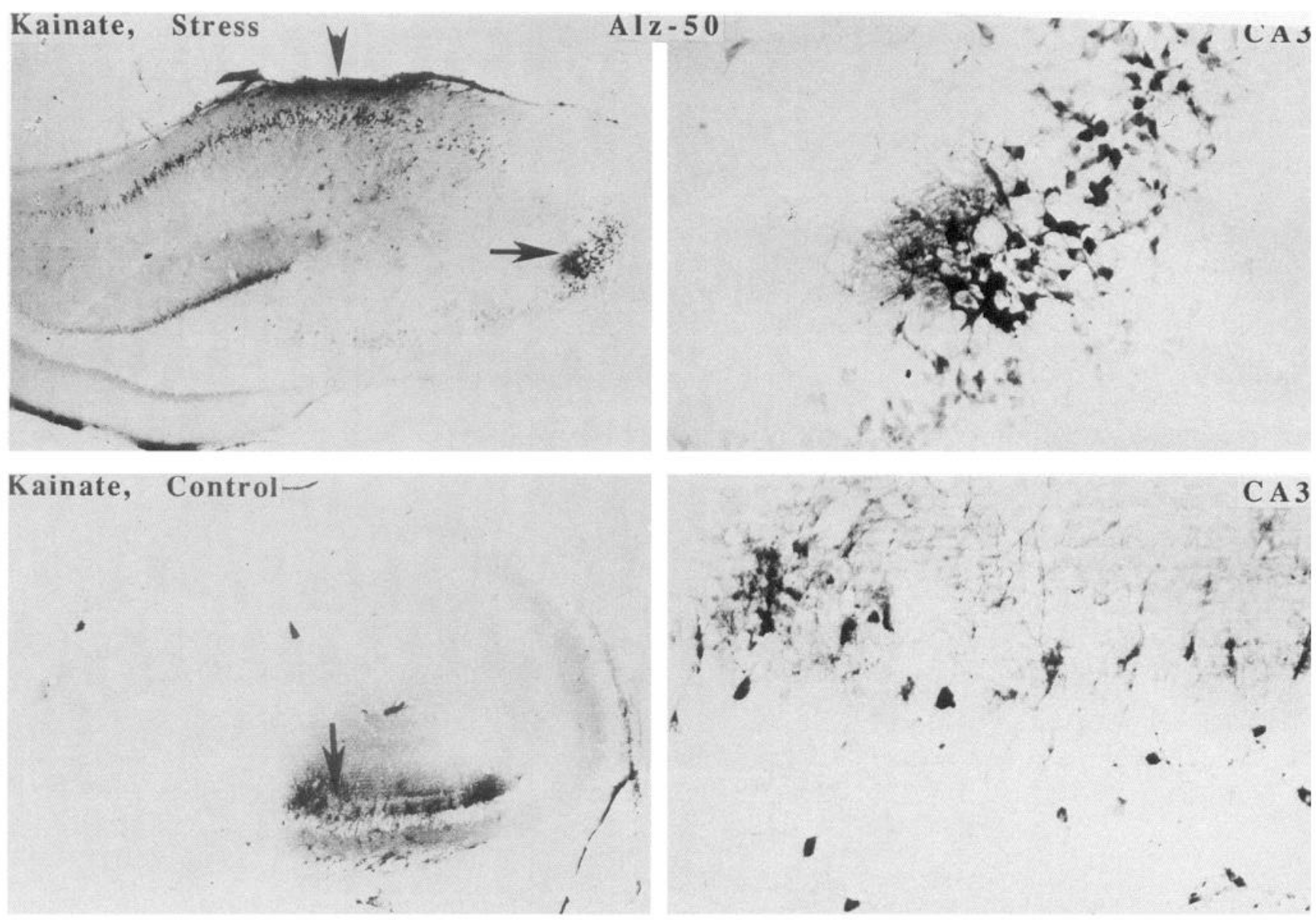

Figure 7. Alz-50 immunoreactivity in hippocampus from KA-injected control and stressed rats. Immunoreactivity is seen in CA3 of both control and stress groups. Immunoreactivity is also seen in CAl of stressed rats but not in controls. The micrographs at the right are higher magnification $(400 \times)$ views of the region of CA3 exhibiting Alz-50 immunoreactivity in the low magnification micrographs $(l e f t ; 40 \times)$. Considerably more neurons are immunoreactive in the stressed rat compared with the unstressed rat.

(Mattson, 1990, 1992; Sautiere et al., 1992). These alterations in tau have been strongly correlated with morphological signs of neuronal injury (Mattson, 1990), and the direct relationship between damaged neurons (as assessed by Nissl staining) and tau immunoreactivity demonstrated in the present study bears this out. Previous work demonstrated loss of MAP2 immunoreactivity associated with ischemic injury to hippocampal neurons (Yanagihara et al., 1990). As with spectrin proteolysis (see below) the damage to MAP2 is believed to result from activation of glutamate receptors, which induces calcium influx and excessive activation of calcium-dependent proteases (Mattson et al., 1988; Siman et al., 1989; Johnson et al., 1991). CORT and stress clearly exacerbated the increased tau immunoreactivity and loss of MAP2 immunoreactivity induced by KA. These data are also consistent with a calcium-mediated mechanism of alterations in tau and MAP2 since CORT enhanced KA-induced calcium mobilization in hippocampal neurons (Elliott et al., 1992, 1993).

Either stress or CORT concentrations in the stress range also exacerbated KA-induced proteolysis of spectrin; this degenerative endpoint was less sensitive to increments in CORT concentrations than was tau immunoreactivity. Kainic acid produces spectrin proteolysis via activation of the calcium-sensitive neutral protease calpain I (Siman et al., 1989). Similar proteolysis occurs following denervation, hypoxia, and global isch- emia (Seubert et al., 1988; Arai et al., 1991). In these cases, the proteolysis is typically most pronounced in moribund neurons, and precedes other indices of degeneration. Furthermore, such proteolysis appears to be intrinsic to the process of neuron death, rather than a mere correlate of it. As evidence, reversal of such proteolysis with calpain inhibitors is neuroprotective (Lee et al., 1991).

In addition to exacerbating neuronal injury to $\mathrm{CA} 3$ neurons, stress unexpectedly promoted degeneration of CA1 neurons in KA-injected hippocampi of approximately $25 \%$ of animals. A corresponding appearance of tau immunoreactivity in the CA1 neurons occurred in these stressed animals. This is of interest because injury to CA1 neurons was not observed in animals receiving the highest dose of CORT pellet, even though these animals had circulating CORT levels at least as high as in the stressed animals. Although this observation will require further characterization, it suggests that stress may affect the vulnerability of hippocampal neurons by a mechanism in addition to elevation of CORT levels. The observation of CA1 damage in stressed animals is also of interest in relation to the possible role of stress and CORT in the pathogenesis of ischemic brain injury and Alzheimer's disease since it is CA1 neurons that are selectively vulnerable in these disorders.

These findings have at least two physiological implications:

(1) Stress, and elevation of CORT concentrations into the 
stress range, worsened the various degenerative endpoints in this study. Profound amounts of $\mathrm{GCs}$ are secreted in response to the stressfulness of insults such as seizure or cardiac arrest, in both humans and expcrimental animals (Feibel et al., 1977; Stein and Sapolsky, 1988). Thus, the GC stress response that accompanies neurological crises is likely to add to the resultant neurodegeneration. Therefore, efforts to attenuate such GC secretion should prove protective; in support of this, administration of metyrapone to rats at the time of the insult decreases or delays the degeneration caused by seizure and hypoxia-ischemia (Stein and Sapolsky, 1988; Morse and Davis, 1990). Furthermore, these data caution against the administration of exogenous GCs following insults involving the hippocampus; the use of GCs at such times to control edema, and the generally poor efficacy of such a practice, has been discussed elsewhere (Sapolsky and Pulsinelli, 1985).

(2) Subtler elevations of CORT concentrations may be neuroendangering as well. Our data indicate that elevation of CORT concentrations from the circadian trough to the peak (i.e., from $0 \%$ to $60 \%$ pellets) exacerbated neuron loss and tau immunoreactivity. This suggests that syndromes associated with elevated GC secretion into the upper basal range can be associated with impaired hippocampal resistance to excitotoxic insults. Such enhanced cortisol secretion occurs in about half the cases of Alzheimer's disease and CORT levels correlate with progression of the disease (Weiner et al., 1993), and this is particularly pertinent in light of the enhanced tau immunoreactivity that is central to the disease's neuropathology (Selkoe, 1991). Furthermore, about half of individuals with major depression secrete elevate concentrations of GCs basally, in some cases even into the Cushingoid range (i.e., $\gtrsim 20 \mu \mathrm{g} / \mathrm{dl}$; APA Taskforce, 1987). Finally, aging in both humans and rodents is associated with elevated basal secretion of GCs (reviewed in Sapolsky, 1990, 1992), and this might play some role in the impaired resistance of the aged hippocampus to neurological insults (discussed in Beal, 1992).

\section{References}

Akana S, Cascio C, Du J, Levin N, Dallman M (1986) Reset of feedback in the adrenocortical system: an apparent shift in sensitivity of adrenocorticotropin to inhibition by corticosterone between morning and evening. Endocrinology 119:2325-2332.

APA Taskforce on Laboratory Test in Psychiatry (1987) The dexamethasone suppression test. An overview of its current status in psychiatry. Am J Psychiatry 144:1253-1264.

Arai H, Passonneau J, Lust W (1986) Energy metabolism in delayed neuron death in CAl neurons of the hippocampus following transient ischemia in the gerbil. Metab Brain Dis 1:263-270.

Armanini M, Hutchins C, Stein B, Sapolsky R (1990) Glucocorticoid endangerment of hippocampal neurons is NMDA receptor-dependent. Brain Res 532:7-13.

Real M (1992) Does impairment of energy metabolism result in excitotoxic neuronal death in neurodegenerative illnesses? Ann Neurol 31:119-129.

Chou Y, Lin W, Sapolsky R (1994) Glucocorticoids increase extracellular $\left[{ }^{3} \mathrm{H}\right] \mathrm{D}$-aspartate overflow in hippocampal cultures during cyanide-induced ischemia. Brain Res, in press.

Elliott E, Sapolsky R (1992) Corticosterone enhances kainic acidinduced calcium mobilizationin cultured hippocampal neurons. $J$ Neurochem 59:1033-1038.

Elliott E, Sapolsky R (1993) Corticosterone impairs hippocampal neuronal calcium regulation: possible mediating mechanisms. Brain Res 602:84-90.

Elliott E, Mattson M, Vanderklish P, Lynch G, Chang I, Sapolsky R (1993) Corticosterone exacerbates kainate-induced alterations in hippocampal tau immunoreactivity and spectrin proteolysis in vivo. $\mathrm{J}$ Neurochem 61:57-67.
Feibel J, Hardi P, Campbell M, Goldstein N, Joynt R (1977) Prognostic value of the stress response following stroke. JAMA 238:1374 1380.

Freo U, Holloway H, Kalogeras K, Rapoport S, Soncrant T (1992) Adrenalectomy or metyrapone-pretreatment abolishes cerebral metabolic responses to the serotonin agonist DOI in the hippocampus. Brain Res 586:256-262.

Gwosdow-Cohen A, Chen C, Besch E (1982) Radioimmunoassay (RIA) of serum corticosterone in rats (41391). Proc Soc Exp Biol 170:2939.

Hall E (1990) Steroids and neuronal destruction or stabilization. In: Ciba Foundation symposium 153, Steroids and neuronal activity, $p$ 206. Chichester: Wiley.

Horner H, Packan D, Sapolsky R (1990) Glucocorticoids inhibit glucose transport in cultured hippocampal neurons and glia. Neuroendocrinology 52:57-64.

Hortnagl H, Berger M, Havelec L, Hornykiewicz O (1993) Role of glucocorticoids in the cholinergic degeneration in rat hippocampus induced by ethylcholine aziridinium (AF64A). J. Neurosci 13:29392945.

Joachim D, Morris J, Selkoe D, Kosik K (1987) Tau epitopes are incorporated into a range of lesions in Alzheimer's disease. J Neuropathol Exp Neurol 46:611-622.

Johnson G, Litersky J, Jope R (1991) Degradation of microtubuleassociated protein 2 and brain spectrin by calpain: a comparative study. J Neurochem 56:1630-1638.

Johnson M, Stone D, Bush L, Hanson G, Gibb J (1989) Glucocorticoids and 3,4-methylenedioxymethamphetamine (MDMA)-induced neurotoxicity. Eur J Pharmacol 161:181-187.

Kadekaro M, Masamori I, Gross P (1988) Local cerebral glucose utilization is increased in acutely adrenalectomized rats. Neuroendocrinology 47:329-337.

Kerr D, Campbell L, Applegate M, Brodish A, Landfield P (1991) Chronic stress-induced acceleration of electrophysiologic and morphometric biomarkers of hippocampal aging. J Neurosci 11:13161320.

Koide T, Wieloch T, Siesjo B (1986) Chronic dexamethasone pretreatment aggravates ischemic neuronal necrosis. J Cereb Blood Flow Metab 6:395-403.

Kosik K, Orecchio L, Binder L, Trojanowsky J, Lee V, Lee G (1988) Epitopes that span the tau molecule are shared with paired helical filaments. Neuron 1:817-825.

Krugers H, Jaarsma D, Korf J (1992) Rat hippocampal lactate efflux during electroconvulsive shock or stress is differentially dependent on entorhinal cortex and adrenal integrity. J Neurochem 58:826-832.

Landfield P, Baskin R, Pitler T (1981) Brain-aging correlates: retardation by hormonal-pharmacological treatments. Science 214:581585.

Lee K, Frank S, Vanderklish P, Arai A, Lynch G (1991) Inhibition of proteolysis protects hippocampal neurons from ischemia. Proc Natl Acad Sci USA 88:7233-7238.

Mattson M (1990) Antigenic changes similar to those seen in neurofibrillary tangles are elicited by glutamate and calcium influx in cultured hippocampal neurons. Neuron 4:105-117.

Mattson M (1992) Effects of microtubule stabilization and destabilization on tau immunoreactivity in cultured hippocampal neurons. Brain Res 582:107-118.

Mattson M, Dou P, Kater S (1988) Outgrowth-regulating actions of glutamate in isolated hippocampal pyramidal neurons. $J$ Neurosci 8:2087-2100.

Meaney M, Aitken D, Bhatnager S, van Berkel C, Sapolsky R (1988) Effect of neonatal handling on age-related impairments associated with the hippocampus. Science 239:766-769.

Miller G, Davis J (1991) Post-ischemic surge in corticosteroids aggravates ischemic damage to gerbil CA1 pyramidal cells. Soc Neurosci Abstr 17:302.4.

Mizoguchi K, Kunishita T, Chui D, Tabira T (1992) Stress induces neuronal death in the hippocampus of castrated rats. Neurosci Lett 138:157-164.

Moghaddam B (1993) Stress preferentially increases extraneuronal levels of excitatory amino acids in the prefrontal cortex: comparison to hippocampus and basal ganglia. J Neurochem 60:1650-1657.

Morse J, Davis J (1990) Regulation of ischemic hippocampal damage in the gerbil: adrenalectomy alters the rate of $\mathrm{CA} 1$ cell disappearance. Exp Neurol 110:86-94. 
Sapolsky R (1985) A mechanism for glucocorticoid toxicity in the hippocampus: increased neuronal vulnerability for metabolic insults. J Neurosci 5:1228-1232.

Sapolsky R (1990) The adrenocortical axis. In: Handbook of the biology of aging, 3d ed (Schneider E, Rowe J, eds). New York: Academic.

Sapolsky R (1992) Do glucocorticoid concentrations rise with age in the rat? Neurobiol Aging 13:171-176.

Sapolsky R, Pulsinelli W (1985) Glucocorticoids potentiate ischemic injury to neurons: therapeutic implications. Science 229:1397-1401.

Sapolsky R, Krey L, McEwen B (1984) Glucocorticoid-sensitive hippocampal neurons are involved in terminating the adrenocortical stress-response. Proc Natl Acad Sci USA 81:6174-6178.

Sapolsky R, Krey L, McEwen B (1985) Prolonged glucocorticoid exposure reduces hippocampal neuron number: implications for aging. J Neurosci 5:1221-1227.

Sapolsky R, Uno H, Rebert C, Finch C (1990) Hippocampal damage associated with prolonged glucocorticoid exposure in primates. J Neurosci 10:2897-2903

Sautiere P-E, Sindou P, Couratier P, Hugon J, Wattez A, Delacourte A (1992) Tau antigenic changes induced by glutamate in rat primary culture model: a biochemical approach. Neurosci Lett 140:206-210.

Selkoe D (1991) The molecular pathology of Alzheimer's disease. Neuron 6:487-498.

Seubert P, Ivy G, Larson J, Lee J, Shahi K, Baudry M, Lynch G (1988) Lesions of entorhinal cortex produce a calain-mediated degradation of brain spectrin in dentate gyrus. I. Biochemical studies. Brain Res 459:226-232.

Siman R, Noszek J, Kegerise C (1989) Calpain I activation is specifically related to excitatory amino acid induction of hippocampal damage. J Neurosci 9:1579-1590.

Stein B, Sapolsky R (1988) Chemical adrenalectomy reduces hippocampal damage induced by kainic acid. Brain Res 473:175-180.

Stein-Behrens B, Elliott E, Miller C, Schilling J, Newcombe R, Sapolsky
R (1992) Glucocorticoids exacerbate kainic acid-induced extracellular accumulation of excitatory amino acids in the rat hippocampus. J Neurochem 58:1730-1735.

Talmi M, Carlier E, Soumireu-Mourat B (1993) Similar effects of aging and corticosterone treatment on mouse hippocampal function. Neurobiol Aging 14:239-245.

Theoret Y, Caldwell-Kenkel J, Krigman M (1985) The role of neuronal metabolic insult in organometal neurotoxicity. Toxicologist $6: 491$.

Tombaugh G, Yang S, Swanson R, Sapolsky R (1992) Glucocorticoids exacerbate hypoxic and hypoglycemic hippocampal injury in vitro: biochemical correlates and a role for astrocytes. J Neurochem 59: 137-142.

Uno H, Tarara R, Else J, Suleman M, Sapolsky R (1989) Hippocampal damage associated with prolonged and fatal stress in primates. J Neurosci 9:1750-1711.

Virgin C, Ha T, Packan D, Tombaugh G, Yang S, Horner H, Sapolsky $R$ (1991) Glucocorticoids inhibit glucose transport and glutamate uptake in hippocampal astrocytes: implications for glucocorticoid neurotoxicity. J Neurochem 57:1422-1428.

Watanabe Y, Gould E, McEwen B (1992) Stress induces atrophy of apical dendrites of hippocampal CA3 ncurons. Hippocampus 2:431438.

Weiner M, Volbach S, Svetlik D, Risser (1993) Cortisol secretion and Alzheimer's disease progression: a preliminary report. Biol Psychiatry 34:158-161.

Wolozin B, Pruchnicki A, Dickson D, Davies P (1986) A neuronal antigen in the brains of Alzheimer's patients. Science 232:648-650.

Woolley C, Gould E, McEwen B (1990) Exposure to excess glucocorticoids alters dendritic morphology of adult hippocampal pyramidal neurons. Brain Res.

Yanagihara T, Brengman J, Mushynski W (1990) Differential vulnerability of microtubule components in cerebral ischemia. Acta Neuropathol 80:499-505. 